\title{
The hydrodynamic effect on silicate scale growth in microscopic flow inferred from numerical simulation
}

\author{
Akihiro MIZUSHIMA $^{1}$, Hitoshi MIKADA ${ }^{1}$ and Junichi TAKEKAWA ${ }^{1}$ \\ ${ }^{1}$ Dept. of Civil and Earth Res. Eng., Kyoto University
}

\begin{abstract}
Mineral scaling is a key process for various geophysical phenomena. Especially it is well known that silica scale is the most difficult one to control. The evaluation of the contribution of the chemical kinetic or the hydrodynamic process on the silica scaling is of importance towards the control of membrane scaling. To meet this goal, we compare the simulation result of the amount and the distribution of silica deposition predicted by the chemical kinetic and the hydrodynamic deposition process and the data from a laboratory or a field experiment. We solve the fluid, temperature and the dissolved silica concentration field by using the lattice Boltzmann method. From our simulation result, it is found that the kinetic process is not sufficient for representing the real silica deposition and that the hydrodynamic effect on growth of silica scale is important for both qualitatively and quantitative evaluations. It is, therefore, necessary to emphasize the physical adhesion should be taken into account for reproducing the process of silica scaling. To further predict the physical adhesion of silica particles, we need to analyze various interaction forces among silica particles in flow, membrane, wall, etc. and to establish the simple silica scale growth microscopically.
\end{abstract}

\section{INTRODUCTION}

The formation of inorganic sparingly-soluble salts, anhydrite and silica from geofluid is a key process for the understanding of geophysical phenomena. Heat extraction from geothermal fluid can cause the precipitation of the minerals dissolved in geothermal fluid. The deposition of the minerals on the wall of wells and in the fractures of the geological reservoir, also known as mineral scaling, is one of the main obstacles to increase the effectiveness of utilization of the geothermal resources. If not controlled properly, it could result in the accumulation of a significant thickness of scale which causes the deterioration of the efficacy of heat exchangers. The cementation of the minerals strongly affects the circulation of hydrothermal systems by choking flow channels. Silica can be extracted from the rock by water-rock chemical reactions in the earth's crust and will be transported toward the surface in the hydrothermal solution. When hydrothermal fluid containing silica ascends toward the earth's surface and subjected to cooling, the solution become super-saturated with silica and precipitation will occur (Martin and Lowell, 2000). Self-sealing, which can keep the permeability of the bedrock around the radioactive waste disposal site low by the formation of silica scale, of importance in the understanding of long-term radionuclide mobility and the safety of deep geological repositories for radioactive waste (Bock et al., 2010).
Silica, in amorphous form, is the one of the most abundant mineral which can precipitate in the geothermal field and is most difficult one to predict and manage in geothermal systems among any mineral scaling due to complex mechanism of their formation.

Oversaturated silica is known to precipitate from aqueous solution either by the direct chemisorption of single silicic acid molecules (monomers) or by polymerizing and forming colloidal particles suspended in the solution. Colloidal particles can subsequently be transported to and attach onto a wall.

We suggest that the formation of amorphous silica scales occur via two mechanisms: (1) the chemical and (2) the physical adhesion. The first one, the chemical adhesion depends on the interaction between silanol groups of silica particle with each other or hydroxyl groups of the wall surface (de Guzmann, 2015). On the other hand, physical adhesion depends on the interaction forces induced by particle-particle or particle-wall interactions; van der Waals forces, Electrical double layer forces, gravity and so on.

The features of the chemical kinetic process taking place at the scaling surface may not provide yet other explanations to the present discrepancies in the understanding of the silica scale formation. Garibaldi (1980) observed higher rates of silica scale growth in areas of low fluid velocity. The stagnation point at the front of an exposed cylinder, for example, showed a high rate of deposition. 
Another study has been reported that a high rate of silica scale growth is observed at the pipe joint of the geothermal production well, where the flow stagnates (Mercado et al., 1989). It has been empirically observed that the fluid flow structure can influence silica scaling. In the several work, the deposition of silica onto a flat plate was modelled numerically (Pott et al., 1996, Brown and Dunstall, 2000). Nevertheless, relatively little reports have been undertaken to investigate hydrodynamic effect on silica scaling.

The aim of this work is to evaluate the contribution of the chemical kinetic or the hydrodynamic process to an amount and a distribution of silica deposition. Those results are compared with data from a laboratory experiment and a field observation.

\section{MODELING}

\section{(1) Lattice Boltzmann method}

We use the lattice Boltzmann method to simulate the fluid flow and the transport of the temperature and the solute. In the lattice Boltzmann model, the two-dimensional nine-directional (D2Q9) lattice Bhatnager-Gross-Krook model (LBGK) is set. In this model, the directions of the discrete velocity are given as

$$
\boldsymbol{c}_{i}=\left\{\begin{array}{cl}
c\{\cos (i \pi / 4), \sin (i \pi / 4)\} & \text { for } i=0,2,4,6 \\
\sqrt{2} c\{\cos (i \pi / 4), \sin (i \pi / 4)\} & \text { for } i=1,3,5,7 \\
(0,0) & \text { for } i=8
\end{array}\right.
$$

where $c=\Delta x / \Delta t$ is the particle speed.

The evolution equation of the particle velocity distribution function $f_{i}(\boldsymbol{x}, t)$ reads

$$
f_{i}(\boldsymbol{x}+\Delta \boldsymbol{x}, t+\Delta t)-f_{i}(\boldsymbol{x}, t)=-\frac{1}{\tau}\left[f_{i}(\boldsymbol{x}, t)-f_{i}^{e q}(\boldsymbol{x}, t)\right],
$$

where $\Delta \boldsymbol{x}$ and $\Delta t$ are the lattice spacing and the time step, respectively. The relaxation time $\tau$ is a parameter which characterizes the constitutive behavior of the considered fluid at a macroscopic level. $f_{i}^{e q}(\boldsymbol{x}, t)$ is the local equilibrium distribution function. In order to recover the correct Navier-Stokes equations, the local equilibrium distribution function is defined as

$$
f_{i}^{e q}=\omega_{i} \rho\left[1+3 \frac{\left(\boldsymbol{c}_{i} \cdot \boldsymbol{u}\right)}{c^{2}}+\frac{9}{2} \frac{\left(\boldsymbol{c}_{i} \cdot \boldsymbol{u}\right)^{2}}{c^{4}}-\frac{3}{2} \frac{\boldsymbol{u}^{2}}{\boldsymbol{c}^{2}}\right],
$$

where $\omega_{i}$ represent the weight factors, $\rho$ is the density and $\boldsymbol{u}$ is the flow velocity vector given as follow:

$$
\rho=\sum_{i=0}^{8} f_{i}(\boldsymbol{x}, t), \boldsymbol{u}=\sum_{i=0}^{8} f_{i}(\boldsymbol{x}, t) \cdot \boldsymbol{c}_{i} .
$$

Table 1 Mass of surface silica deposition $24 \mathrm{~h}$ after initiation of calculation (Mizushima et al., 2014)

\begin{tabular}{lll}
\hline Hosoi and Imai (1982) & $0.42-1.7$ & $\mathrm{~g} / \mathrm{m}^{2}$ \\
Chemical kinetic process & $5.0 \times 10^{-5}$ & $\mathrm{~g} / \mathrm{m}^{2}$ \\
Hydrodynamic Process & 11.0 & $\mathrm{~g} / \mathrm{m}^{2}$ \\
\hline
\end{tabular}

\section{(2) Scheme of silica scale formation}

There have been a number of experimental studies made on the chemical kinetics of silica polymerization, which have measured the disappearance of monomeric silica during the course of the reaction (Rimstidt and Barnes, 1980; Bohlmann et al., 1980; Weres et al., 1982; Bird et al., 1986; Fleming, 1986). For generality and simplicity, we consider the general first-order kinetic-reaction model at the fluid-solid interface (Rimstidt and Barnes, 1980);

$$
\frac{\partial C}{\partial t}=-k\left(C-C_{s}\right)
$$

where $C$ is the solute concentration at the interface, $C_{s}$ is the saturation concentration and $k$ is the rate constant.

On the other hand, we consider the physical adhesion of silica particles on the wall surface in flow. The silica scale growth model is used by

$$
\frac{\partial C}{\partial t}=\psi\left(S^{-0.630}\right) C
$$

where $S$ is the shear rate near the wall. $\psi$ is the coefficient determined according to Gentile et al. (2008). Gentile et al. (2008) formulated the relation between the shear rate near the wall and the number of the $1 \mu \mathrm{m}$ spherical silica particles adhered on the wall.

\section{SIMULATIONS AND RESULTS}

To compare the chemical kinetic and the hydrodynamic process of silica scale formation with experimental data, the silica scale formation based on each process is simulated in two models: (1) the parallel channel model and (2) the sudden expansion channel model.

\section{(1) Parallel channel model}

The initial condition of this model is based on the experiment of Hosoi and Imai (1984). The model is set as follows: The channel width; $10 \mathrm{~mm}$, the length; $150 \mathrm{~mm}$. The average flow velocity is 0.01 $\mathrm{m} / \mathrm{s}$, the dissolved silica concentration is $500 \mathrm{ppm}$, fluid temperature is $95{ }^{\circ} \mathrm{C}$, while the outer wall temperature is $25{ }^{\circ} \mathrm{C}$. Table. 1 shows the mass of 


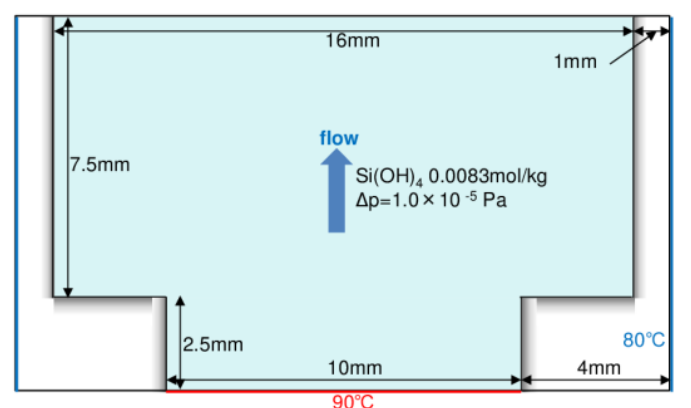

Figure 1 Sudden expansion channel model (initial condition)

surface deposition estimated by both the chemical kinetic and the hydrodynamic process $24 \mathrm{~h}$ after initiation of calculation and obtained by the experiment of Hosoi and Imai (1984). We found that the mass of silica deposition predicted by the chemical kinetic process provides extremely lower magnitude of the deposition than that of laboratory experiment conducted by Hosoi and Imai (1984). Silica deposition predicted by the hydrodynamic process shows the similar magnitude as that observed by Hosoi and Imai (1984) although this hydrodynamic process model has not been accurately reproduced the condition of their experiment.

\section{(2) Sudden expansion channel model}

This model is used to reproduce a high rate of silica scale growth where the flow stagnates predicted by the chemical kinetic or the hydrodynamic process (Figure 1). Figure 2 shows the time change of the build-up of silica scale based on the hydrodynamic process. The rate of the build-up of silica scale is so slow that only the deposition rate is accelerated at $10^{5}$ the speed of the real speed in order to predict the scale in an acceptable computational time. As the result, the scale form estimated by the hydrodynamic process tend to be consistent with that of the production-wall pipe of the geothermal well observed by Mercado et al. (1989) where the flow stagnates and at the downstream-side wall of the sudden expansion.

\section{DISCUSSION}

According to our simulation result, the amount of the silica scale predicted by the hydrodynamic process somewhat differ from that of the experiment conducted by Hosoi and Imai (1984), but such differences can be considered to reflect the differences in the conditions of the real silica scale growth process, which has not been accurately understood, as well as differences in the condition

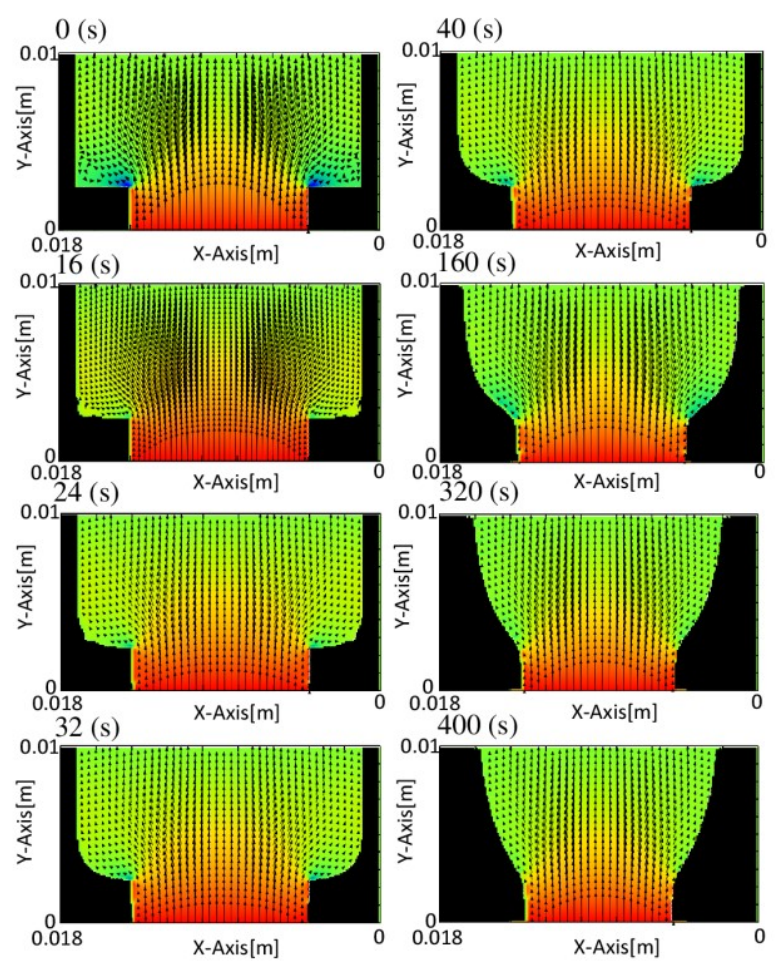

Figure 2 Time change of build-up of silica scale at $0 \mathrm{~s}, 16 \mathrm{~s}, 24 \mathrm{~s}, 32 \mathrm{~s}, 40 \mathrm{~s}, 160 \mathrm{~s}, 320 \mathrm{~s}$ and $400 \mathrm{~s}$ after initial of calculation. Black arrows; flow velocity and color contour; pressure difference.

of the experiments of Gentile et al. (2008) which refer to the experimental scale growth rate law. Gentile et al. (2008) analyzes the margination of leukocytes by using $1 \mu \mathrm{m}$ silica particles in laminar flow. The fluid viscosity and the wall friction in our simulation models are not consistent with those set by Gentile et al. (2008). We assume that the number of the dissolved silica adhered on the wall is equal to that of the $1 \mu \mathrm{m}$ spherical silica particles, although the real silica particles is 10 to $100 \mathrm{~nm}$ (Kokhanenko et al., 2012). These differences render the results to be qualitative rather than quantitative. Especially, it is suggested that the physical adhesion is strongly affected by particle size effect (Brown and Dunstall, 2000).

To further predict the physical adhesion of silica particles, we need to analyze the interaction force on each silica particle and the particle motion in flow and to establish the simple silica scale growth microscopically, which is to help us to establish the model of the silica scale growth in channel flow by the feedback from the simple constitutive law of the microscopic analysis.

The physics of transport, deposition, detachment and re-entrainment of colloidal particles or nanoparticles suspended in a fluid are important in many areas of fluids engineering, e.g. fouling of 
heat exchangers, contamination of nuclear reactors, plugging of filtration membranes and occlusion of human veins, deposits in microelectronics and in the paper industry. A number of works have been simulated to analyze these phenomena, and it has been numerically and experimentally shown that surface interactions of the following interaction forces can play a significant role in clogging mechanisms.

- DLVO forces

In the absence of additives such as polymeric dispersants and coagulants, van der Waals force and electrostatic force are considered to be the dominant forces in the particle suspensions. The effect of these forces is commonly modelled using the well-known DLVO theory, which is the algebraic summation of van der Waals attractive force and electrostatic repulsive force.

- Brownian forces

Brownian diffusion effect, also referred to as molecular diffusion, becomes the dominant transport mechanism for particles less than $500 \mathrm{~nm}$ and is especially significant as the particles become smaller. The Brownian random force was often modelled as a Gaussian white noise process.

Other forces that will be considered are the buoyancy force, the drag and lift forces, the near the-wall hydraulic retardation and the Brownian. These are the forces considered to have an influence on the dynamics of colloidal particles or nanoparticles. Therefore, they need to be incorporated in the momentum conservation equation of the discrete element modelling.

\section{CONCLUSIONS}

We developed a physicochemical model including both chemical kinetic and hydrodynamic processed for correctly predicting overall silica scale formation. A numerical simulation code including the general first-order kinetic-reaction model at the fluid-solid interface based on the lattice Boltzmann method was developed to see how scale would grow with various interaction forces. Our numerical simulation results led us to conclude that the hydrodynamic effect on the growth of silica scale is larger than the chemical kinetic process and is important for both qualitative and quantitative evaluations. We would like to emphasize that the physical adhesion of silica particles should be take into account for reproducing the growth of silica scale. Furthermore, we should include the other interaction forces acting on silica particles in our simulation to analyze the motion of each silica particle more precisely.

\section{REFERENCES}

1) Bird, G., Boon, J., and Stone, T., 1986, Silica transport during steam injection into oil sands I. Dissolution and precipitation kinetics of quartz -new results and review of existing data, Chemical Geology, 54, 69-80.

2) Bock, H., Dehandschutter, B., Martin, C. D., Mazurek, M., de Haller, A., Skoczylas, F., Davy, C., 2010, Self-sealing of fractures in Argillaceous Formations in the context of geological disposal of radioactive waste- review and synthesis, Report no. 6184, Nuclear Energy Agency.

3) Bohlmann, E. G., Mesmer R. E., and Berlinski, P., 1980, Kinetics of silica deposition from simulated geothermal brines, Journal of Petroleum Science and Engineering, 20, 239-248.

4) Brown, K, and Dunstall, M., 2000, Silica scaling under controlled dynamic conditions, Proceedings World Geothermal Congress 2000, 3039-3044

5) Fleming, B. A., 1986, Kinetics of reaction between silicic acid and amorphous silica surfaces in $\mathrm{NaC} 1$ solutions, Journal of Colloid and Interface Science, 110, 40-64.

6) Garibaldi, F., 1980, The effect of some hydrodynamic parameters on silica deposition. Diploma Project 80.11, Geothermal Institute, University of Auckland.

7) Gentile, F., Chiappini, C., Fine, D., Bhavane, R. C., Peluccio, M. S., Ming-Cheng Cheng, M., Liu, X., and Decuzzi, P., 2008, The effect of shape on the margination dynamics of non-neutrally buoyant particles in two-dimensional shear flows, Journal of Biomechanics, 41, 2312-2318.

8) de Guzman, R. C., Christopher, C. A., Tiu, B. D. B., Baltazar Jr., A. D., and Rigoberto, C., 2015, Characterization of Nanocolloidal Silica Formation of Untreated and Treated Simulated Geothermal Brine through Various Particle Size and Zeta Potential Measurement Techniques, Proceedings World Geothermal Congress 2015

9) Hosoi, M., and Imai, H., 1982, Study on precipitation and prevention of the silica scale form the geothermal hot water, Journal of the Geothermal Research Society of Japan, 4, 127-142

10) Kokhanenko, P., Masuri, S., Jermy, M., Sellier, M., and Brown, K., 2012, Hydrodynamics and electrochemistry of silica scaling, Proceedings New Zealand Geothermal Workshop 2012.

11) Martin, T., and Lowell, P., 2000, Precipitation of quartz during high-temperature, fracture-controlled hydrothermal upflow at 
ocean ridges: Equilibrium versus linear kinetics, Journal of Geophysical Research, 105, no. B1, 869-882.

12) Mercado, M., Bermejo, F., Hurtado, R., Terrazas, B. and Hernandez, L., 1989, Scale incidence of production pipes of Cerro Prieto geothermal wells, Geothermics, 18, no. 1/2, 225-232.

13) Mizushima, A., Mikada, H., Goto, T. and Takekawa, J., 2014, Fluid-physical simulation of silicate scale growth using lattice Boltzmann method, Expanded Abstruct Society of Exploration Geophysicists, p. 3025-3029.

14) Rimstidt, J. D., and Barnes, H. L., 1980, The kinetics of silica-water reactions, Geochimica et Cosmochimica Acta, 44, 1683-1699.

15) Weres, D., A. Yee, and Tsao, L., 1982, Equations and type curves for predicting the polymerisation of amorphous silica in geothermal brines, Journal of Petroleum Science and Engineering, 22, 9-16. 